\title{
ON ASYMPTOTICALLY STABLE SETS
}

\author{
by D. DESBROW \\ (Received 21st November 1969)
}

\section{Introduction}

In this paper we study closed sets having a neighbourhood with compact closure which are positively asymptotically stable under a flow on a metric space $X$. For an understanding of this and the rest of the introduction it is sufficient for the reader to have in mind as an example of a flow a system of first order, autonomous ordinary differential equations describing mathematically a time-independent physical system; in short a dynamical system. In a flow a set $M$ is positively stable if the trajectories through all points sufficiently close to $M$ remain in the future in a given neighbourhood of $M$. The set $M$ is positively asymptotically stable if it is positively stable and, in addition, trajectories through all points of some neighbourhood of $M$ approach $M$ in the future.

It is hardly surprising that asymptotic stability in flows, having its origin in classical dynamics, has been intensively studied. The reader is referred to Bhatia and Szegö's book (2) for a fairly complete account. A large number of characterisations of asymptotic stability, some free from the concept of stability, are in the literature. Here we present three new ones, each "stability-free" and expressed, essentially, in terms of the behaviour in the past of trajectories outside the set.

It is no real restriction as we shall see and it facilitates exposition if we confine our attention in the rest of the introduction to a closed subset $M$ of a flow, having a neighbourhood with compact closure and positively invariant in the sense that trajectories through all points of $M$ lie in $M$ in the future. Our result establishes that each of the following conditions is equivalent to the positive asymptotic stability of $M$ :

(i) $M$ has a neighbourhood outside of which all trajectories, other than those in $M$, lie ultimately in the past;

(ii) $M$ has a neighbourhood containing no negative prolongation limit points of points outside $M$;

(iii) $M$ has a neighbourhood containing no negative limit points of points outside $M$.

A point $y \in X$ is a negative prolongation limit point of the point $x \in X$ if there are points $x^{*}, y^{*} \in X$ arbitrarily close to $x, y$ respectively with $y^{*}$ on the trajectory through $x^{*}$ arbitrarily far in the past. A special case occurs when $x^{*}$ can be taken to be $x$. Then $y$ is a negative limit point of $x$. 
According to the first equivalence positive asymptotic stability, itself a type of "strong " positive stability, can be characterised by that type of "strong" negative instability which is condition (i). The second equivalence compares favourably with the characterisation of positively stable sets as those sets $M$ containing no negative prolongation limit points of points outside $M$-an almost immediate consequence of the definitions. On the other hand the third equivalence is in sharp contrast with the situation for positively stable sets, which cannot be characterised as those sets $M$ containing no negative limit points of points outside $M$. See, for example, Ura ((7), § 3, p. 257).

\section{Definitions and notation}

In what follows $X$ is a metric space. If $M \subseteq X$ and $N \subseteq X, \bar{M}$ denotes the closure of $M$ and $N-M$ the complement of $M$ in $N$. If $M \subseteq X$ and for each $x \in X, L(x)$ is a subset of $X$ then $L(M)$ denotes the set $\cup\{L(x): x \in M\}$.

Let $\mathscr{T}$ denote either the additive group $\mathbf{R}$ of real numbers with the usual topology or the additive group $\mathbf{Z}$ of integers with the discrete topology and let $\pi$ be a continuous map from $X \times \mathscr{T}$ into $X$ such that for each $x \in X$ and each $s, t \in \mathscr{T}$

$$
\pi(x, 0)=x, \quad \pi(\pi(x, s), t)=\pi(x, s+t) .
$$

Then $(X, \mathscr{T}, \pi)$ is called a flow on $X$ and is said to be continuous or discrete according as $\mathscr{T}$ is $\mathbf{R}$ or $\mathbf{Z}$. For $x \in X$ and $t \in \mathscr{T}$ it is more convenient to write $x t$ for $\pi(x, t)$ if no confusion can arise.

For $x \in X$ the set $\gamma(x)=\{x t \in X: t \in \mathscr{T}\}$ is called the trajectory of $x$ and the set $\gamma^{+}(x)=\{x t \in \gamma(x): t \geqq 0\}$ is called the positive semi-trajectory of $x$. Let $M \subseteq X$ and $x \in X$. Then the positive semi-trajectory $\gamma^{+}(x)$ is said to be ultimately outside $M$ if $\gamma^{+}(x \tau) \cap M=\varnothing$ for some $\tau \geqq 0$.

A non-void set $M \subseteq X$ is said to be invariant if $\gamma(M)=M$ and positively invariant if $\gamma^{+}(M)=M$. A closed, invariant set is said to be minimal if it has no proper, closed, invariant subset. A compact invariant set always contains a minimal set.

A set $M \subseteq X$ is said to be isolated from minimal sets if it has a neighbourhood containing no minimal sets except those (if any) in $M$.

For $x \in X$ the positive prolongation limit set of $x$, denoted $J^{+}(x)$, is the set of all points $y \in X$ for which there exist sequences $\left\{x_{n}\right\}$ in $X$ and $\left\{t_{n}\right\}$ in $\mathscr{T}$ with $x_{n} \rightarrow x, t_{n} \rightarrow+\infty$ and $x_{n} t_{n} \rightarrow y$. For $x \in X$ the positive (or omega) limit set of $x$, denoted $L^{+}(x)$, is the set of all points $y \in X$ for which there exists a sequence $\left\{t_{n}\right\}$ in $\mathscr{T}$ with $t_{n} \rightarrow+\infty$ and $x t_{n} \rightarrow y$. Both $J^{+}(x)$ and $L^{+}(x)$ are closed, invariant sets and clearly $L^{+}(x) \subseteq J^{+}(x)$ for all $x \in X$.

Throughout the present paragraph $M$ shall denote a non-void, closed subset of $X$ having a neighbourhood with compact closure. The set $M$ is said to be positively stable if for any neighbourhood $U$ of $M$ there is a neighbourhood $V$ of $M$ such that $\gamma^{+}(V) \subseteq U$. Otherwise $M$ is said to be positively unstable. If $M$ is positively stable and in addition there is a neighbourhood $U$ 
of $M$ such that $L^{+}(U) \subseteq M, M$ is said to be positively asymptotically stable. The set $M$ is said to be a positive uniform attractor if there is a neighbourhood $U$ of $M$ and, for any neighbourhood $V$ of $M$, a $\tau \geqq 0$ such that $\gamma^{+}(U \tau) \subseteq V$. For the type of set considered here the definitions of stability and asymptotic stability are equivalent to the usual definitions occurring in the literature, in particular " $\varepsilon, \delta$ " definitions, and are more convenient for our purposes. The reader is warned that Bhatia and Szegö use the term uniform attractor in a sense different to ours.

Corresponding " negatively sensed" definitions can be formulated throughout in an obvious manner. Notations are sensed by affixing to them the appropriate + or - sign.

The reader is referred to Chapter 2 of Bhatia and Szegö's book (2) for a general account of continuous flows on metric spaces, including most of the concepts defined here.

\section{An auxiliary result}

In this section we establish two characterisations of asymptotic stability which we shall need later. Firstly, however, it is necessary to list the following known facts concerning stability.

Lemma. Let $M$ be a non-void, closed subset of $X$ having a neighbourhood with compact closure. Then

(i) if $M$ is positively stable it is positively invariant and $J^{-}(X-M) \cap M=\varnothing$;

(ii) if $M$ is positively invariant and $J^{+}(M) \subseteq M$ then $M$ is positively stable;

(iii) if $M$ is positively invariant, isolated from minimal sets and

$$
L^{-}(X-M) \cap M=\varnothing
$$

then $M$ is positively stable.

(i) and (ii) are elementary. (iii) follows from Theorem 3 of (4) if it be observed that the proof as given applies if $M$ is merely positively invariant rather than invariant.

We now give the auxiliary result.

Theorem 1. Let $M$ be a non-void, closed subset of $X$ having a neighbourhood with compact closure. Then the following are pairwise equivalent:

(i) $M$ is positively asymptotically stable;

(ii) $M$ is a positively invariant, positive uniform attractor;

(iii) $M$ is positively stable and isolated from minimal sets.

For continuous flows that positive asymptotic stability implies positive uniform attraction is due to Lefschetz ((5), (19.2)). For a proof in flows see Lemma 1 of (3) and observe that the invariance of $M$ there assumed is irrelevant to the proof given. This together with the above lemma shows that (i) implies (ii). 
Bhatia, Lazer and Szegö ((1), Theorem 3.7) have proved the equivalence of (i) and (ii) for continuous flows when $X$ is locally compact and $M$ is compact. They use definitions which are trivially equivalent to ours and their proof applies under present hypotheses on $M$. It needs adapting, however, for discrete flows and so we prefer to prove here that (ii) implies (i).

Proof (ii) $\Rightarrow$ (i). Suppose $M$ is a positively invariant, positive uniform attractor. Clearly there is a sequence $\left\{V_{k}\right\}$ of neighbourhoods of $M$ with compact closure such that $M=\cap \bar{V}_{k}$.

Firstly $M$ is positively stable. Let $y \in J^{+}(M)$. Then there exists a point $x \in M$ and sequences $\left\{x_{n}\right\}$ in $X,\left\{t_{n}\right\}$ in $\mathscr{T}$ with $x_{n} \rightarrow x, t_{n} \rightarrow+\infty$ and $x_{n} t_{n} \rightarrow y$. Since $M$ is a positive uniform attractor there is a neighbourhood $U$ of $M$ and for any $k, \tau_{k} \geqq 0$ such that $\gamma^{+}\left(U \tau_{k}\right) \subseteq V_{k}$. Further for any $k$ and all sufficiently large $n, x_{n} \in U$ and $t_{n} \geqq \tau_{k}$ so that $x_{n} t_{n} \in \gamma^{+}\left(x_{n} \tau_{k}\right) \subseteq \gamma^{+}\left(U \tau_{k}\right) \subseteq V_{k}$. Thus for all $k, y \in \bar{V}_{k}$ so that $y \in \cap \bar{V}_{k}=M$. Hence $J^{+}(M) \subseteq M$ and since $M$ is positively invariant it is positively stable.

Secondly $L^{+}(U) \subseteq M$. Let $u \in U$. Then for any $k, \gamma^{+}\left(u \tau_{k}\right) \subseteq V_{k}$ and so $L^{+}(u) \subseteq \overline{\gamma^{+}\left(u \tau_{k}\right)} \subseteq \bar{V}_{k}$. Hence $L^{+}(u) \subseteq \cap \bar{V}_{k}=M$ and so $L^{+}(U) \subseteq M$.

It follows that $M$ is positively asymptotically stable.

It is immediate that (i) implies (iii). Saito ((6), Theorem 5) has shown that (iii) implies (i) for continuous flows when $M$ is compact and $X$ is locally compact. His proof applies under present assumptions when the flow is continuous but depends on the theory of saddle sets which he develops. Here we give a short, alternative proof valid for flows.

Proof (iii) $\Rightarrow$ (i). Suppose $M$ is positively stable and isolated from minimal sets. Then there is a neighbourhood $U$ of $M$ whose closure is compact and contains no minimal sets not contained in $M$. Further there is a neighbourhood $V$ of $M$ such that $\gamma^{+}(V) \subseteq U$. If $M$ is not positively asymptotically stable there exists $p \in V$ and $L^{+}(p) \nsubseteq M$. Now $L^{+}(p) \subseteq \overline{\gamma^{+}(p)} \subseteq \bar{U}$ so that $L^{+}(p)$ is compact and thus contains a minimal set, necessarily in $M$. Hence $L^{+}(p) \cap M \neq \varnothing$. Now there exists $r \in L^{+}(p)-M \subseteq J^{+}(p)$. Thus $p \in J^{-}(r)$ and $L^{+}(p) \subseteq J^{-}(r)$ so that $\varnothing \neq L^{+}(p) \cap M \subseteq J^{-}(r) \cap M$. It follows from the above lemma that $M$ is positively unstable; a contradiction in view of which $M$ is positively asymptotically stable.

\section{Characterisations of asymptotic stability}

We now state and prove the main result of the paper.

Theorem 2. Let $M$ be a non-void, closed subset of $X$ having a neighbourhood with compact closure. Then the following are pairwise equivalent:

(1) $M$ is positively asymptotically stable;

(2) $M$ is positively invariant and there is a neighbourhood $U$ of $M$ such that any negative semi-trajectory outside $M$ is ultimately outside $U$; 
(3) $M$ is positively invariant and there is a neighbourhood $V$ of $M$ such that $J^{-}(X-M) \cap V=\varnothing$

(4) $M$ is positively invariant and there is a neighbourhood $W$ of $M$ such that $L^{-}(X-M) \cap W=\varnothing$.

Proof (1) $\Rightarrow$ (2). Suppose $M$ is positively asymptotically stable. Since by the previous theorem $M$ is necessarily isolated from minimal sets, there is a neighbourhood $U^{*}$ of $M$ such that, as we may suppose, $U^{*}$ is compact and there are no minimal sets in $\overline{U^{*}}$ other than those in $M$. Since $M$ is positively stable there is a neighbourhood $U$ of $M$ such that $\gamma^{+}(U) \subseteq U^{*}$ and, as we may suppose, $U \subseteq U^{*}$. Now for any $x \in X-M$ either $\gamma^{-}(x) \cap U^{*}=\varnothing$ or $\gamma^{-}(x) \cap U^{*} \neq \varnothing$. In the former case clearly $\gamma^{-}(x)$ is ultimately outside $U$. In the latter case there exists $y \in \gamma^{-}(x) \cap U^{*}$. We show that $\gamma^{-}(y) \notin U^{*}$. For if not $\varnothing \neq L^{-}(y) \subseteq \overline{\gamma^{-}(y)} \subseteq \overline{U^{*}}$ and $L^{-}(y)$ being compact contains a minimal set, necessarily in $M$. Hence $L^{-}(y) \cap M \neq \varnothing$. Since

$$
\varnothing \neq L^{-}(X-M) \cap M \subseteq J^{-}(X-M) \cap M,
$$

according to the lemma, $M$ is positively unstable; a contradiction in view of which $\gamma^{-}(y) \notin U^{*}$. Thus there exists $z \in \gamma^{-}(y)-U^{*}$ and so by the definition of $U, \gamma^{-}(z) \cap U=\varnothing$. Thus in this case also $\gamma^{-}(x)$ is ultimately outside $U$.

Now $M$, being positively stable, is positively invariant and the implication is proved.

Proof (1) $\Rightarrow(3)$. Let $M$ be positively asymptotically stable. Since by the previous theorem $M$ is necessarily a positive uniform attractor there is an open neighbourhood $V$ of $M$ and for any neighbourhood $V^{*}$ of $M$ a $\tau \geqq 0$ such that $\gamma^{+}(V \tau) \subseteq V^{*}$. We show that $J^{-}(X \backslash M) \cap V=\varnothing$. For if not there exist $x \in V, y \in X-M$ with $x \in J^{-}(y)$ or equivalently $y \in J^{+}(x)$. Thus there exist sequences $\left\{x_{n}\right\}$ in $X$ and $\left\{t_{n}\right\}$ in $\mathscr{T}$ with $x_{n} \rightarrow x, t_{n} \rightarrow+\infty$ and $x_{n} t_{n} \rightarrow y$. Clearly we may suppose that $x_{n} \in V$ for all $n$. Since $y \notin M$ there is a neighbourhood $V^{*}$ of $M$ such that $y \notin \overline{V^{*}}$. Thus there exists $\tau \geqq 0$ such that $\gamma^{+}(V \tau) \subseteq V^{*}$. Clearly we may suppose that $t_{n} \geqq \tau$ for all $n$. Then

$$
x_{n} t_{n} \in \gamma^{+}\left(x_{n} \tau\right) \subseteq \gamma^{+}(V \tau) \subseteq V^{*}
$$

so that $y \in \overline{V^{*}} ;$ a contradiction in view of which $J^{-}(X-M) \cap V=\varnothing$.

As above, $M$ is necessarily positively invariant and so the implication is proved.

That (2) and (3) both imply (4) (with $W$ the interior of $U$ and $V$ respectively) is clear enough not to require proof here.

Proof (4) $\Rightarrow(1)$. Let $M$ be positively invariant and have a neighbourhood $W$ such that $L^{-}(X-M) \cap W=\varnothing$. A fortiori, $L^{-}(X-M) \cap M=\varnothing$. We show that $M$ is isolated from minimal sets. Clearly we may assume $\bar{W}$ compact. Let $N$ be a necessarily compact, minimal subset of $W$. Then $L^{-}(N)=N \subseteq W$ 
so that $N \subseteq M$. Thus $M$ is isolated from minimal sets. It follows from the lemma that $M$ is positively stable and being isolated from minimal sets, positively asymptotically stable according to the previous theorem.

This completes the proof of the theorem.

\section{REFERENCES}

(1) N. P. Bhatta, A. C. Lazer and G. P. Szegö, On global weak attractors in dynamical systems, J. Math. Anal. Appl. 16 (1966), 544-552.

(2) N. P. BhatiA and G. P. SzEgö, Dynamical systems: stability theory and applications, Lecture notes in mathematics no. 35 (Springer-Verlag, Berlin-HeidelbergNew York, 1967).

(3) D. Desbrow, On connexion, invariance and stability in certain flows, Proc. Cambridge Philos. Soc. 60 (1964), 51-55.

(4) D. DesBrow, On unstable invariant sets, Funkcial. Ekvac. 13 (1970), 109-126.

(5) S. LeFsChETz, Liapunov and stability in dynamical systems, Bol. Soc. Mat. Mexicana (2) 3 (1958), 25-39.

(6) T. SArto, On a compact invariant set isolated from minimal sets, Funkcial. Ekvac. 12 (1969), 193-203.

(7) T. URA, On the flow outside a closed, invariant set; stability, relative stability and saddle sets, Contributions to Differential Equations, 3 (1964), 249-294.

UNIVERSITY OF EDINBURGH

EDINBURGH EH1 $1 \mathrm{HZ}$ 